\title{
Band-offset driven efficiency of the doping of SiGe core-shell nanowires
}

\author{
R. Rurali, M. Amato, and S. Ossicini
}

\begin{abstract}
Impurity doping of semiconducting nanowires is expected to become increasingly inefficient as the wire diameter shrinks down, because impurity states get deeper due to quantum and dielectric confinement. Here we show that efficient $n$ - and p-type doping can be achieved in strongly confined SiGe core-shell nanowires, taking advantage of the type-II band offset at the $\mathrm{Si} / \mathrm{Ge}$ interface. $\mathrm{A}$ one-dimensional electron (hole) gas is created at the band-edge and the carrier density is uniquely controlled by the impurity concentration with no need of thermal activation. Additionally, SiGe coreshell nanowires provide naturally the separation between the different types of carriers, electron and holes, and are ideally suited for photovoltaic applications.
\end{abstract}

\section{INTRODUCTION}

$\mathrm{O}$ $\mathrm{NE}$ of the main limit of doping of pure silicon and pure germanium nanowires ( $\mathrm{Si}$ and $\mathrm{Ge}$ NWs) is its inefficiency when the diameter is reduced, as a consequence of surface segregation of impurities, strong quantum confinement and dielectric mismatch [1-2]. In the case of doping with boron or phosphorus impurities of $\mathrm{Si}$ and $\mathrm{Ge}$ NWs the impurity state is deep into the band gap and cannot be electrically activated at typical device temperatures. This phenomenon is responsible of several problems related to the real applications of these types of materials as electronic devices.

Manuscript received June 13, 2012. M.A. thanks the Transnational Access Programme of the HPC-EUROPA2 Project. S.O. acknowledges the European Community's Seventh Framework Programme (FP7/2007-2013) under Grant Agreement 245977, MIUR-PRIN 2007, Ministero Affari Esteri, Direzione Generale per la Promozione and Cooperazione Culturale and Fondazione Cassa di Risparmio di Modena. Funding under Contract Nos. TEC2009-06986, FIS2009-12721-C04-03, and CSD2007-00041 are greatly acknowledged.

Michele Amato was with Dipartimento di Scienze e Metodi dell'Ingegneria and Centro Interdepartmentale En\&Tech, Università di Modena e Reggio Emilia, Via Amendola 2 Pad. Morselli, I-42100 Reggio Emilia, Italy. He is now with the Laboratoire des Solides Irradiés, École Polytechnique, 91128 Palaiseau, France and Université Paris Sud, Laboratoire de Physiques des Solides, 91405 Orsay, France (e-mail: michele.amato@unimore.it).

Stefano Ossicini is with the Dipartimento di Scienze e Metodi dell'Ingegneria and Centro Interdepartmentale En\&Tech, Università di Modena e Reggio Emilia, Via Amendola 2 Pad. Morselli, I-42100 Reggio Emilia, Italy (e-mail: stefano.ossicini@unimore.it).

Riccardo Rurali is with the Institut de Ciència de Materials de Barcelona (CSIC), Campus de Bellaterra, 08193 Bellaterra, Spain (phone: +34 93 5801853; e-mail: rrurali@icmab.es).
The reason for the deepening of the impurity state is that in a strongly confined regime the spacing between electronic states increases, following the standard particle-in-a-box idealized model, leading to larger activation energy of the dopant. On top of that, even when the effects of quantum confinement vanish, it has been shown that the impurity state is still deeper than in the bulk material due to the so-called dielectric confinement [2]. Finally, dopant agents added during the nanowire growth are more stable close to the surface, where the host lattice strain is more easily released. There, however, dopants can combine with surface defects such as dangling bonds, leading to electrically inactive complexes $[3,4]$.

All these issues make the doping of a onedimensional nanoscale semiconductor challenging and the possibility of engineering devices based on sub-10 nm doped nanowires should be seriously questioned. In the following, we present theoretical calculations, showing how some of these problems can be easily overcome by opportune doping with boron and phosphorus impurities in core-shell silicon-germanium NWs [5].

\section{Computational Details}

We have performed first-principles electronic structure calculations within density-functional theory, as implemented in the SIESTA package [6]. We have used norm-conserving pseudopotentials of the Troullier-Martins type for the core electrons [7] and have expanded the one-electron wave function of the valence electrons with an optimized double- $\zeta$ basis set plus polarization functions [8]. The exchange-correlation energy is calculated within the local density approximation (LDA). We consider core-shell NWs grown along the [110] orientation with a diameter of 
approximately $2.4 \mathrm{~nm}$ (core $\sim 1.4 \mathrm{~nm}$ ). The shell surface dangling bonds have been saturated with hydrogen atoms, as it customary in this type of calculations. In order to neglect the spurious interaction with the periodic images of the impurity, we have used supercells made of six NW primitive cells [9]. All the systems discussed have been structurally relaxed with a standard conjugate gradient algorithm until all the forces on the atoms were lower than $0.04 \mathrm{eV} / \AA$. The large size of the computational cell allows us to sample the Brillouin zone with the $\Gamma$ point only, though we have recalculated the electronic structure of the relaxed geometries with a finer grid of $(1 \times 1 \times 16)$ k-points.

\section{RESUlTS AND DISCUSSION}

In this work we focus on the core doping of Gecore Si-shell and Si-core Ge-shell NWs. As it has been previously shown, in these nanostructures, the band offset between the two materials causes localization of the valence states on germanium and of conduction states on silicon [10]. As a consequence of this property, with particular doping conditions, a one-dimensional electron (hole) gas at the band edge can be created and the carrier density is uniquely controlled by the impurity concentration with no need of thermal activation.

Let us start considering the case of Ge-core Sishell NWs, which are the most common SiGe coaxial structures grown [11]. If the core is doped with a $\mathrm{P}$ substitutional impurity, the energy required to activate its carrier into the $\mathrm{Ge}$ conduction band is too large for typical device operation temperature. This is what would happen for a stand-alone Ge NW with a diameter of the order of the core of the SiGe coaxial wire that we are discussing. However, when the $\mathrm{Si}$ shell is deposited its conduction (and valence) band will line up according to the type-II band alignment described in Ref.[10] and the P electron can fall into the lower energy Si-shell conduction band. This will happen as long as the offset between the core and shell conduction band is larger than the depth of the $\mathrm{P}$ impurity electronic state. This situation is illustrated in Figure 1 where we display the calculated band structure diagram. The impurity state, whose wave-function is shown in the inset, lies some $0.2 \mathrm{eV}$ below the Ge core states, but is well within the Si shell empty states, so that its carrier can fall down to the bottom of the conduction band. This mechanism gives rise to the formation of an electron gas, whose signature is the fact that the Fermi level is not pinned by the defect state, as in conventional impurity doping, but is rather lined-up with the conduction band edge.

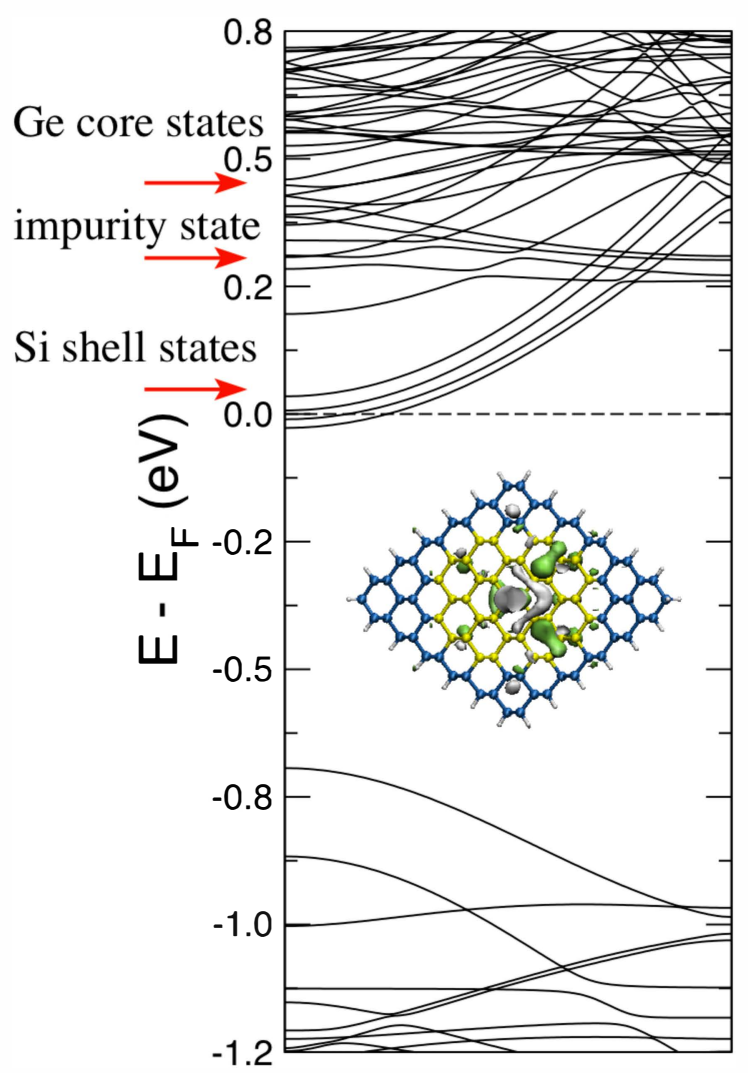

Figure 1. Band structure of a $\mathrm{Ge}_{\text {core }} / \mathrm{Si}_{\text {shell }} \mathrm{NW}$ with $\mathrm{P}$ doping in the core Arrows indicate the lowest lying core and shell states and the impurity state whose real-space distribution is shown in the inset. Doping of the Ge core yields an electron at the bottom of the conduction band where the Fermi level is pinned.

A similar trend can be observed for the less common Si-core Ge-shell geometry [12]. When doping the $\mathrm{Si}$ core with $\mathrm{B}$, the impurity state would be to far from the Si valence band to accept an electron, i.e. to create a hole. Nevertheless, in presence of a Ge shell, with a higher energy valence band upon type-II band alignment, the formation of a hole is always possible and does not even need to be thermally activated. Again, Figure 2 shows the bands of the coaxial wire, where the impurity state lie within Ge-shell states, allowing the formation of a hole gas. Here the 
Fermi level is at the valence band edge, where the hole coming from the B impurity ends up.

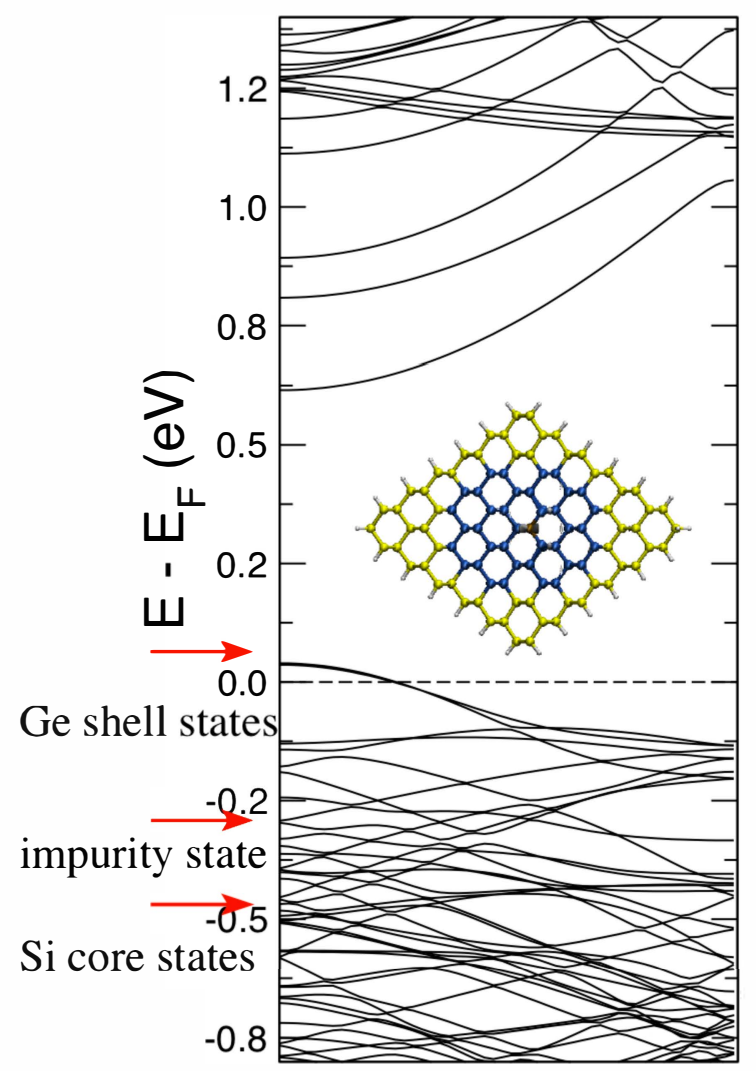

Figure 2. Band structure of a $\mathrm{Si}_{\text {core }} / \mathrm{Ge}_{\text {shell }} \mathrm{NW}$ with $\mathrm{B}$ doping in the core. Arrows indicate the highest lying core and shell states and the impurity state whose real-space distribution is shown in the inset. Doping of the Si core yields a hole at the top of the valence band where the Fermi level is pinned.

Notice that both these efficient $n$ - and $p$-type core doping mechanisms share the peculiarity that while impurity are added into the core, the electron or hole gas are created in the wire shell.

It should be stressed that while band-offset allows us getting rid of the undesired consequences of quantum confinement, the presence of a shell deposited after the axial growth of the core minimizes the effect of surface segregation -if the impurities diffuse towards the interface, the deposition of the shell prevent at a large extent their passivation with defects - and dielectric confinement -as the core and the shell have dielectric constant of the same order $\left(\varepsilon_{\mathrm{Si}} \sim 11.9\right.$, $\left.\varepsilon_{\mathrm{Ge}} \sim 16\right)$.

The formation of a hole gas in SiGe core-shell NWs have been experimentally observed, in agreement with our predictions [13]. Electron gas, on the other hand, has not been reported yet. We believe that the reason is that in wires currently grown, the confinement size is such that the required band-offset for the formation of the carrier gas has already vanished for electrons, while it still survives for holes.

\section{CONCLUSION}

We have presented first-principles calculations of the electronic structure of doped SiGe coreshell nanowires. Type-II band offset, where conduction states are localized on $\mathrm{Si}$, while valence states are localized in $\mathrm{Ge}$, has been proposed to increase the efficiency of SiGe nanostructures for photovoltaic applications, as it provides a natural mechanism for effective charge separation. In this work we have shown that additionally, SiGe band alignment increases the efficiently of dopants such as B and P that would otherwise be too deep in strongly confined onedimensional nanostructures.

\section{REFERENCES}

[1] R. Rurali, "Structural, electronic, and transport properties of silicon nanowires," Rev. Mod. Phys. 82, 427 (2010).

[2] M. Diarra, Y.-M. Niquet, C. Delerue, and G. Allan, "Ionization energy of donor and acceptor impurities in semiconductor nanowires: Importance of dielectric confinement," Phys. Rev. B 75, 045301 (2007).

[3] M. V. Fernández-Serra, Ch. Adessi, and X. Blase, "Surface Segregation and Backscattering in Doped Silicon Nanowires," Phys. Rev. Lett. 96, 166805 (2006).

[4] M. V. Fernández-Serra, Ch. Adessi, and X. Blase, "Conductance, Surface Traps, and Passivation in Doped Silicon Nanowires," Nano Lett. 6, 2674 (2006).

[5] M. Amato, S. Ossicini, and R. Rurali, "Band-Offset Driven Efficiency of the Doping of SiGe Core-Shell Nanowires," Nano Lett. 11, 594 (2011).

[6] José M. Soler, Emilio Artacho, Julian D. Gale, Alberto García, Javier Junquera, Pablo Ordejón, and Daniel Sánchez-Portal, "The Siesta method for ab initio order-N materials simulation," J. Phys.: Condens. Matter 14, 2745 (2002)

[7] N. Troullier and J. L. Martins, "Efficient pseudopotentials for planewave calculations," Phys. Rev. B 43, 1993 (1991).

[8] E. Artacho, D. Sánchez-Portal, P. Ordejón, A. García, and J. M. Soler, "Linear-scaling ab-initio calculations for large and complex systems (with a brief review of applications)," Phys. Stat. Sol. (b) 215, 809 (1999).

[9] R. Rurali, M. Palummo, and X. Cartoixà, "Convergence study of neutral and charged defect formation energies in Si nanowires," Phys. Rev. B 81, 235304 (2010).

[10] M. Amato, M. Palummo, and S. Ossicini, "Reduced quantum confinement effect and electron-hole separation in SiGe nanowires," Phys. Rev. B 79, 201302(R) (2009).

[11] K.-K. Lew, L. Pan, E. C. Dickey, J. M. Redwing, "Vapor-LiquidSolid Growth of Silicon-Germanium Nanowires," Adv. Mater. 15, 2073 (2003).

[12] L. Pan, K.-K. Lew, J. M. Redwing, and E. C. Dickey, "StranskiKrastanow Growth of Germanium on Silicon Nanowires," Nano Lett. 5, 1081 (2005). 
[13] W. Lu, J. Xiang, B. P. Timko, Y. Wu, and C. M. Lieber, "Onedimensional hole gas in germanium/silicon nanowire heterostructures," Proc. Natl. Acad. Sci. USA 102, 10046 (2005). 